\title{
Synthetic stellar and SSP libraries as templates for Gaia simulations
}

\author{
Rosanna Sordo • Antonella Vallenari • Rosaria Tantalo • France Allard • \\ Ronny Blomme · Jean-Claud Bouret • Ines Brott • Yves Fremat • \\ Christophe Martayan · Yassine Damerdji • Bengt Edvardsson · Eric Josselin · \\ Bertrand Plez • Oleg Kochukhov • Mary Kontizas • Ulisse Munari • Tenay Saguner • \\ Jean Zorec • Andreas Schweitzer • Paraskevi Tsalmantza
}

Received: 16 September 2009 / Accepted: 8 January 2010 / Published online: 5 February 2010

(C) Springer Science+Business Media B.V. 2010

\author{
R. Sordo $(\varangle) \cdot$ A. Vallenari $\cdot$ U. Munari $\cdot$ T. Saguner \\ INAF-Osservatorio Astronomico di Padova, Padua, Italy \\ e-mail: rosanna.sordo@oapd.inaf.it \\ R. Tantalo $\cdot$ T. Saguner \\ Astronomy Department, Padova University, Padua, Italy \\ F. Allard \\ CRAL-ENS, Lyon, France \\ R. Blomme · Y. Fremat · C. Martayan · J. Zorec \\ Royal Observatory, Brussels, Belgium \\ J.-C. Bouret \\ CNRS, Marseille, France \\ I. Brott \\ Sterrenkundig Instituut Utrecht, Utrecht, The Netherlands \\ Y. Damerdji \\ Univ. De Liege, Liege, Belgium \\ B. Edvardsson \\ Mid Sweden University, Sundsvall, Sweden \\ E. Josselin · B. Plez \\ GRAAL, Montpellier, France \\ O. Kochukhov \\ Uppsala University, Uppsala, Sweden \\ M. Kontizas \\ Athens University, Athens, Greece \\ A. Schweitzer \\ Hamburger Sternwarte, Hamburg, Germany \\ P. Tsalmantza \\ MPI für Astronomie, Heidelberg, Germany
}

\begin{abstract}
ESA's Gaia mission will collect low resolution spectroscopy in the optical range for $\sim 10^{9}$ objects. Complete and up-to-date libraries of synthetic stellar spectra are needed to built algorithms aimed to automatically derive the classification and the parametrization of this huge amount of data. In addition, libraries of stellar spectra are one of the main ingredients of stellar population synthesis models, aiming to derive the properties of unresolved stellar populations from their integrated light. We present (a) the newly computed libraries of synthetic spectra built by the Gaia community, covering the whole optical range (300$1100 \mathrm{~nm})$ at medium-high resolution of $(0.3 \mathrm{~nm})$ for stars spanning the most different types, from $\mathrm{M}$ to $\mathrm{O}$, from Apeculiar to Emission lines to White Dwarfs, and (b) the implementation of those libraries in our SSP code (Tantalo in The Initial Mass Function 50 Years Later, 327:235 2005), exploring different stellar evolution models.
\end{abstract}

Keywords Gaia · Stars · Synthetic spectroscopy · Population synthesis

\section{Introduction}

ESA's Gaia mission, to be launched early 2012, will obtain accurate position, parallax and proper motion for $10^{9} \mathrm{ob}-$ jects all over the sky, up to magnitude $G=20(V=20-22)$ with an astrometric accuracy at the $\mu$-arcsec level, providing in addition low-dispersion spectroscopy for each object and radial velocity (up to $G=16$ ). The final catalog is expected to provide the discrete classification of sources (single stars, galaxies, QSOs, asteroids) and the astrophysical parameters (APs) for single stars (i.e. $T_{\text {eff }}, \log g, \ldots$ ) and possibly the parametrization of special sources (galaxies). Gaia will produce an unprecedented amount of data (40-50 GB/day) 
Table 1 Synthetic stellar libraries for Gaia. The first set of libraries is already implemented in the present work, the inclusion of the remaining is ongoing

\begin{tabular}{|c|c|c|c|c|}
\hline Name & $T_{\text {eff }}(\mathrm{K})$ & $\log g$ & {$[\mathrm{Fe} / \mathrm{H}]$} & Notes \\
\hline Munari $^{\mathrm{a}}$ & $3500-47500$ & $0.0-5.0$ & $-2.5-+1.0$ & Kurucz models, $[\alpha / \mathrm{Fe}]=+0.0,+0.4 ;$ Rotational velocities explored; \\
\hline $\mathrm{O}, \mathrm{B}$ stars $^{\mathrm{b}}$ & $15000-50000$ & $1.0-5.0$ & $-5.0-+1.0$ & TLUSTY code, NLTE, wind, mass loss \\
\hline $\mathrm{Ap} / \mathrm{Bp} \operatorname{stars}^{\mathrm{c}}$ & $7000-16000$ & 4.0 & +0.0 & LLmodels code, non-solar abundance \\
\hline B-F stars ${ }^{\mathrm{d}}$ & $6000-16000$ & $2.5-4.5$ & +0.0 & LLmodels \\
\hline MARCS $^{\mathrm{e}}$ & $4000-8000$ & $-0.5-5.5$ & $-5.0-+1.0$ & Variations in individual $\alpha$-elements abundances \\
\hline C stars ${ }^{\mathrm{e}, \mathrm{f}}$ & $4000-8000$ & $0.0-5.0$ & $-5.0-+0.0$ & $\Delta T_{\text {eff }}=500 \mathrm{~K} ;[\mathrm{C} / \mathrm{Fe}]=0,1,2,3 ;[\alpha / \mathrm{Fe}]=+0.0,+0.4$ \\
\hline PHOENIXg & $3000-10000$ & $-0.5-5.5$ & $-3.5-+0.5$ & $\Delta T_{\text {eff }}=100 \mathrm{~K} ;[\alpha / \mathrm{Fe}]=-0.2-+0.8, \Delta[\alpha / \mathrm{Fe}]=0.2$ \\
\hline Ultra-cool dwarfs ${ }^{\mathrm{h}}$ & $100-6000$ & $0.0-6.0$ & +0.0 & Different dust model implemented \\
\hline $\mathrm{Be}^{\mathrm{i}}$ & $15000-25000$ & 4.0 & +0.0 & Different envelope radius considered \\
\hline $\mathrm{WR}^{\mathrm{i}}$ & $25000-51000$ & $2.8-4.0$ & +0.0 & Different mass loss rate considered \\
\hline$W D^{j}$ & $6000-90000$ & $7.0-9.0$ & & WDA \& WDB, LTE \\
\hline $\mathrm{sdOB}^{\mathrm{k}}$ & $26000-100000$ & $4.8-6.4$ & +0.00 & NLTE, $\Delta T_{\text {eff }}=500 \mathrm{~K}, \Delta \log g=0.2$ \\
\hline
\end{tabular}

References: ${ }^{\mathrm{a}}$ Munari et al. (2005), ${ }^{\mathrm{b}}$ Bouret et al. (2008), ${ }^{\mathrm{c}}$ Kochukhov and Shulyak (2008), ${ }^{\mathrm{d}}$ Shulyak et al. (2004), ${ }^{\mathrm{e}}$ Gustafsson et al. (2008), ${ }^{\mathrm{f}}$ Alvarez and Plez (1998), ${ }^{\mathrm{g}}$ Brott and Hauschildt (2005), ${ }^{\mathrm{h}}$ Allard et al. (2000), ${ }^{\mathrm{i}}$ Martayan et al. (2008), ${ }^{\mathrm{j}}$ Castanheira et al. (2006), ${ }^{\mathrm{k}}$ Hindson et al. (2008)

leading to $100 \mathrm{~TB}$ of compressed data in 5 years. This huge number of observed objects must be classified in an automated way. Those data will be classified using supervised methods (Bailer-Jones et al. 2008) by comparison with a set of suitable templates. Large and up-to-date libraries of stellar spectra are thus of fundamental importance in the Gaia era, as training data for the algorithms to interpret the observational data. A large effort is ongoing (thanks to the Gaia community) to cover as much as possible the HR diagram at the best of the present knowledge, producing synthetic datasets from the cooler stars to the faster evolutionary stages. In addition, coupling the spectrum of a star (at all possible evolutionary stages) with evolutionary stellar models will allow one to derive the properties of unresolved stellar populations from their integrated light (we refer among others to Fioc and Rocca-Volmerange 1997; Vallenari and Sordo 2008; Tsalmantza et al. 2007, 2009). Here we present the use of the Gaia stellar libraries to calculate Single Stellar Populations (SSPs) as building blocks toward galaxy population synthesis.

\section{Synthetic stellar libraries}

The Gaia community agreed to provide synthetic stellar libraries, calculated with an homogeneous coverage of parameter space at $0.3 \mathrm{~nm}$ resolution in the optical range (300$1100 \mathrm{~nm}$ ). These new libraries, summarized in Table 1, span a large range in atmospheric parameters with great uniformity in the parameter space coverage and resolution. An unprecedented accuracy in the reproduction of the observed stellar spectra is also ensured: the different codes are optimized to the $T_{\text {eff }}$ regimes, providing an adequate treatment for all the physical processes relevant in the stellar atmosphere, from very cool to hot, from the most metal rich to very metal poor stars: NLTE effects, dust models for the very cool stars, the effect of mass loss, emission from circumstellar envelopes, variation of single element chemical abundances, magnetic field, $\alpha$-enhancement (see Table 1 for the references). These libraries are described in more detail in Sordo et al. (2009), together with their high resolution version (covering the CaII triplet region, at $0.001 \mathrm{~nm}$ sampling).

\section{Single stellar population}

Gaia will observe $10^{7}$ unresolved galaxies. SSPs are an essential tool to model and interpret the spectral energy distribution of clusters and galaxies. For a given age and metallicity, first theoretical stellar models are used to compute the distribution of stars along the isochrones, following an Initial Mass Function, then the appropriate stellar spectrum is assigned to each star and summed up to obtain the integrated light.

Together with the stellar library calculated for Gaia, the Munari et al. (2005) synthetic stellar library was also used in the preparation of the final algorithms especially for the radial velocities determination. Based on Kurucz's codes, the library covers the whole HR diagram with great uniformity, allowing one to investigate the effects of $[\alpha / \mathrm{Fe}]$ variations up to relatively high $T_{\text {eff }}$. This library (in its solar-scaled version) was extended in the UV, using the UVBlue library 
Fig. 1 Comparison between our SSP library with $Z=0.01$, $[\alpha / \mathrm{Fe}]=+0.4$ (red dots) and the SSP library presented by (Coelho et al. 2007, blue solid line), for a sample of Lick indexes sensitive to $\alpha$-enhancement. The units are $\AA$ for $\mathrm{Ca} 4455$ and $\mathrm{Mgb}$ indexes, mag for $\mathrm{Mg} 1$ and $\mathrm{Mg} 2$ indexes
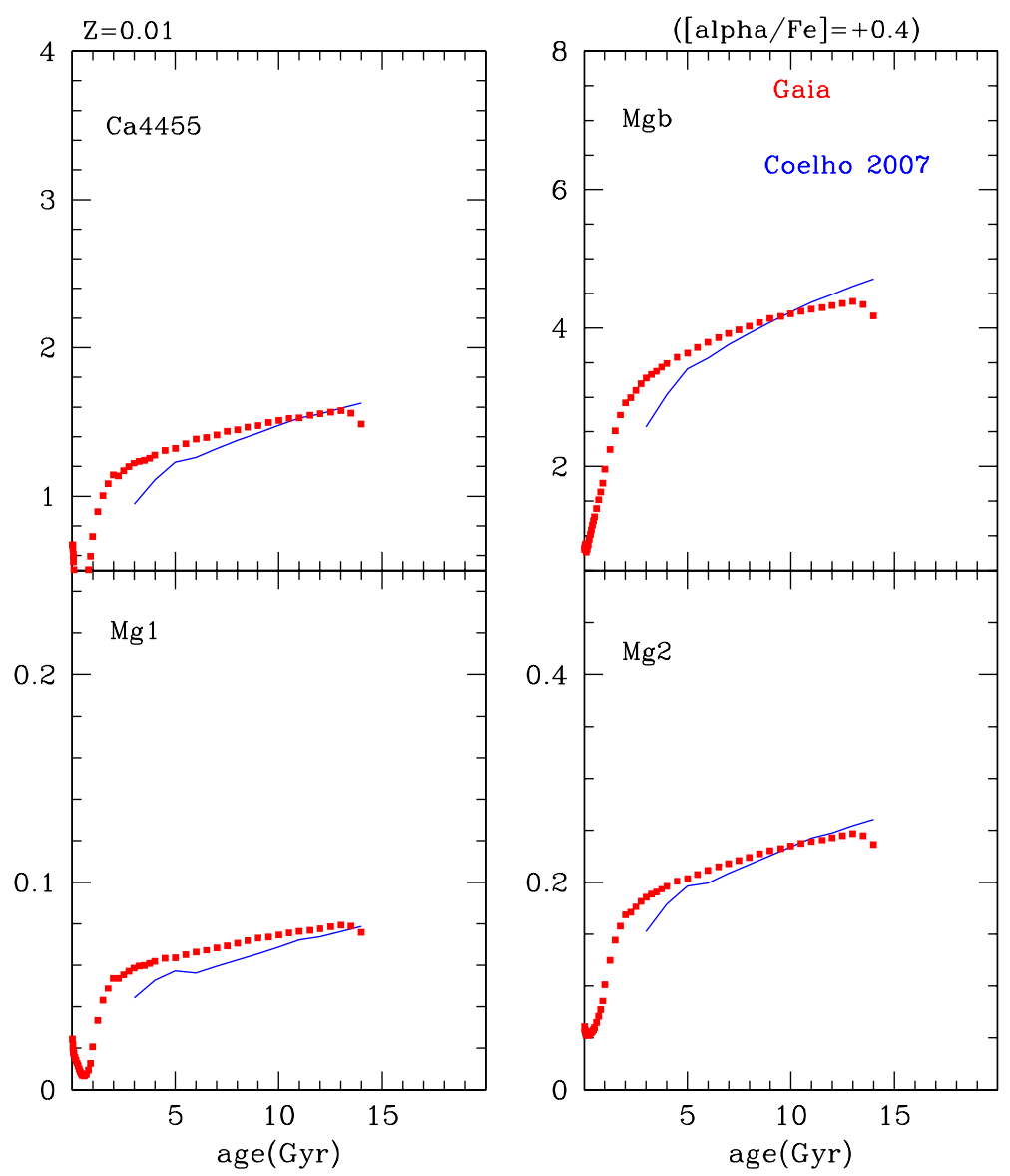

(Rodríguez-Merino et al. 2005), which uses Kurucz's model atmospheres and covers the $85-470 \mathrm{~nm}$ spectral range.

Using the code presented by Tantalo (2005) we produced a different SSP dataset, coupling different synthetic stellar libraries and theoretical models:

1. UV + Optical solar-scaled SSPs: we used Padova isochrones by Bertelli et al. (1994) together with the Munari et al. library extended by UVBlue library. The SSPs have $[\alpha / \mathrm{Fe}]=+0.0$.

2. Optical solar-scaled SSPs: the Padova isochrones by Bertelli et al. (1994) are coupled with the Gaia libraries presented in Table 1 . The SSPs have $[\alpha / \mathrm{Fe}]=+0.0$.

3. Optical $\alpha$-enhanced SSPs: Teramo isochrones by Pietrinferni et al. (2006) are coupled with the Munari et al. library. The SSPs have $[\alpha / \mathrm{Fe}]=+0.4$.

The implementation of the set of isochrones by Marigo et al. (2008) is on-going.

The final datasets can be implemented into galaxy evolutionary codes (i.e. assuming a Star Formation Rate and a chemical evolution model) to study the integrated light of unresolved galaxies.

Extensive tests on the results (Lick index calculation, comparison with observed clusters) will be presented in Vallenari et al. (2010). In Fig. 1, we show the comparison of a few Lick indexes calculated from our SSP library ( $\alpha$ enhanced version) with the results presented by Coelho et al. (2007). In Fig. 2, Lick indexes are compared with LMC clusters data. Index values, ages and metallicity of the clusters are taken from Beasley et al. (2002).

\section{Conclusions}

Refined high resolution stellar spectra are of fundamental importance in the Gaia era, to train the algorithms used to interpret the large volume of observational data. We present the libraries of synthetic spectra that have been calculated for the Gaia mission.

We implemented the Gaia synthetic libraries into our SSP code, to produce three different datasets according to the different evolutionary model adopted and to the $\alpha$-enhancement. An example of the comparison with Lick indexes published in the literature is shown, while more detailed results will be presented in Vallenari et al. (2010). The SSPs can be implemented into galaxy evolutionary codes to study the integrated light of unresolved populations. 
Fig. 2 Comparison between a few SSP Lick indexes with $Z=0.008$ (red) and $Z=0.0004$ (green), $[\alpha / \mathrm{Fe}]=+0.0$ and observational data of LMC clusters as function of cluster age (see text for details). The units are $\AA$ for $\mathrm{Ca} 4455, \mathrm{Mgb}$ and $\mathrm{H}_{\beta}$ indexes, mag for $\mathrm{Mg} 1$ and $\mathrm{Mg} 2$ indexes
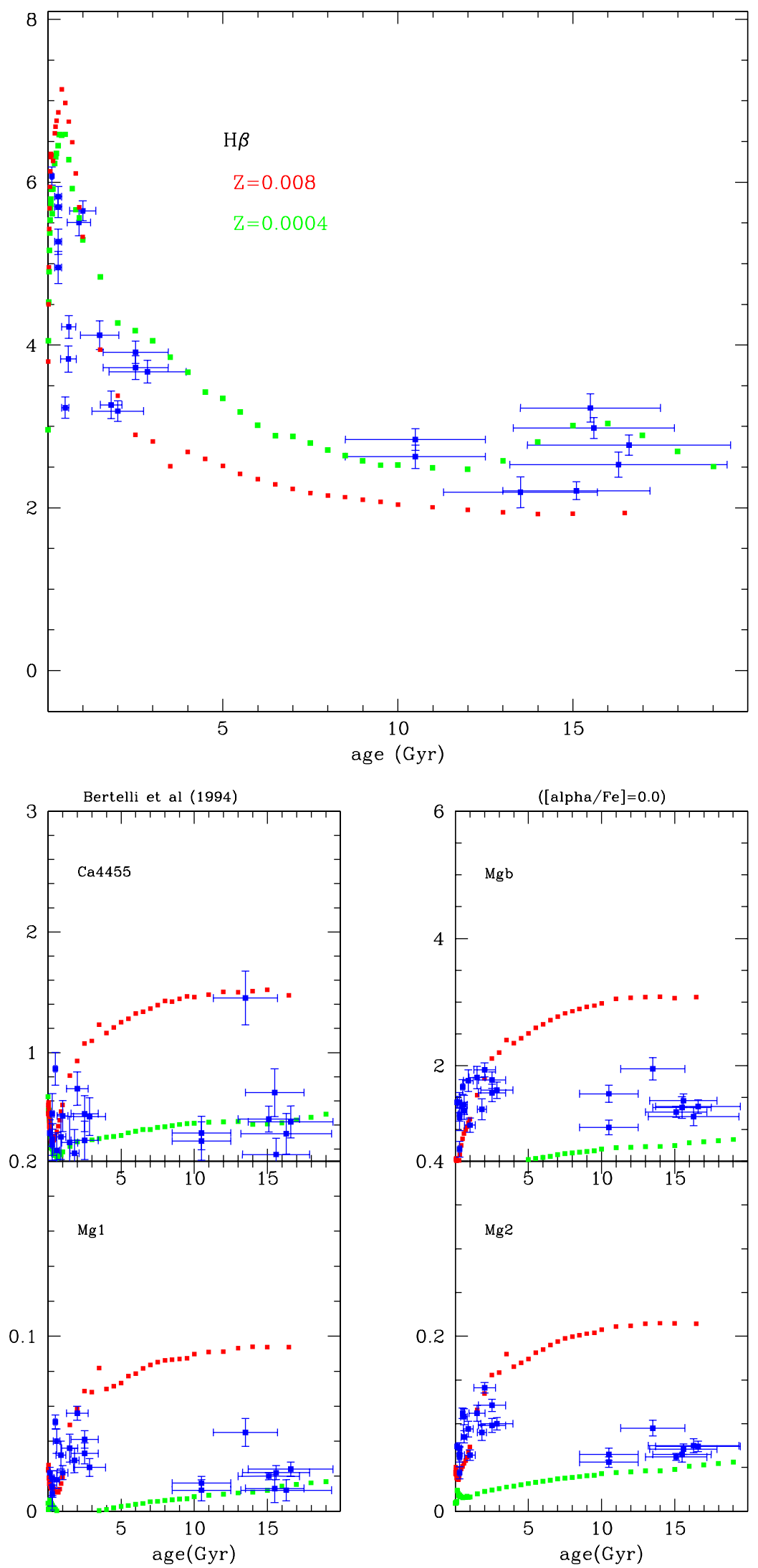


\section{References}

Alvarez, R., Plez, B.: Astron. Astrophys. 330, 1109 (1998)

Allard, F., Hauschildt, P.H., Schweitzer, A.: Astrophys. J. 539, 366 (2000)

Beasley, M.A., Hoyle, F., Sharples, R.M.: Mon. Not. R. Astron. Soc. 336, 168 (2002)

Bailer-Jones, C.A.L., Smith, K.W., Tiede, C., Sordo, R., Vallenari, A.: Mon. Not. R. Astron. Soc. 391, 1838 (2008)

Bertelli, G., Bressan, A., Chiosi, C., Fagotto, F., Nasi, E.: Astron. Astrophys. Suppl. Ser. 106, 275 (1994)

Bouret, J.-C., et al.: Rev. Mex. Astron. Astrofis. Conf. Ser. 33, 50 (2008)

Brott, I., Hauschildt, P.H.: The three-dimensional universe with Gaia, 576, 565 (2005)

Castanheira, B.G., et al.: Astron. Astrophys. 450, 331 (2006)

Coelho, P., Bruzual, G., Charlot, S., Weiss, A., Barbuy, B., Ferguson, J.W.: Mon. Not. R. Astron. Soc. 382, 498 (2007)

Gustafsson, B., Edvardsson, B., Eriksson, K., Jørgensen, U.G., Nordlund, Å., Plez, B.: Astron. Astrophys. 486, 951 (2008)

Fioc, M., Rocca-Volmerange, B.: Astron. Astrophys. 326, 950 (1997)
Hindson, L., Napiwotzki, R., Heber, U., Lemke, M.: Hot subdwarf stars and related objects. ASP Conf. Ser. 392, 163 (2008)

Kochukhov, O., Shulyak, D.: Contrib. Astron. Obs. Skalnaté Pleso 38, 419 (2008)

Marigo, P., Girardi, L., Bressan, A., Groenewegen, M.A.T., Silva, L., Granato, G.L.: Astron. Astrophys. 482, 883 (2008)

Martayan, C., et al.: In SF2A-2008, p. 499 (2008)

Munari, U., Sordo, R., Castelli, F., Zwitter, T.: Astron. Astrophys. 442, 1127 (2005)

Pietrinferni, A., Cassisi, S., Salaris, M., Castelli, F.: Astrophys. J. 642, 797 (2006)

Rodríguez-Merino, L.H., Chavez, M., Bertone, E., Buzzoni, A.: Astrophys. J. 626, 411 (2005)

Shulyak, D., et al.: Astron. Astrophys. 428, 993 (2004)

Sordo, R., Vallenari, A., et al.: Mem. Soc. Astron. Ital. 80, 103 (2009)

Tantalo, R.: The initial mass function 50 years later. Astrophys. Space Sci. Libr. 327, 235 (2005)

Tsalmantza, P., et al.: Astron. Astrophys. 470, 761 (2007)

Tsalmantza, P., et al.: arXiv:0907.1671 (2009)

Vallenari, A., Sordo, R.: arXiv:0812.0293 (2008)

Vallenari, A., et al.: (2010) in preparation 